\title{
The Darboux Point ${ }^{1}$
}

\author{
P. M. Mereau ${ }^{2}$ and W. F. Powers ${ }^{3}$ \\ Communicated by ]. V. Breakwell \\ Dedicated to Professor A. Busemann
}

\begin{abstract}
A theory of global optimality based upon the Darbouxpoint concept is developed. A definition is proposed for the Darboux point, and the Darboux point is shown to exist on nonglobally optimal trajectories under relatively general conditions. A mutually exclusive classification of Darboux points is noted, and several properties are proved for one of these classes (the Type-1 Darboux point). Numerous examples are included to illustrate the Darbouxpoint definition and properties.
\end{abstract}

Key Words. Calculus of variations, optimal control, global sufficient conditions, Darboux point, conjugate point.

\section{List of Symbols}

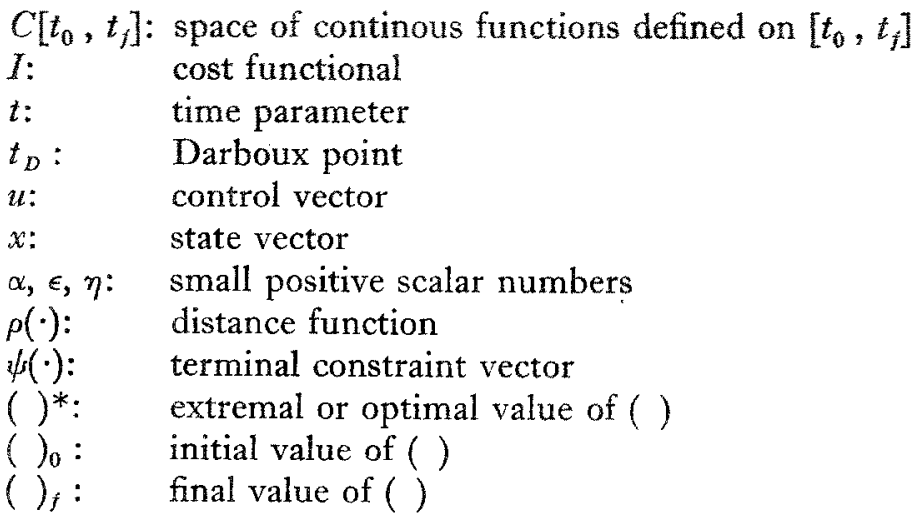

1 This research was supported by the National Science Foundation under Grant No. GK-30115.

2 Engineex, ADERSA/GERBIOS, Vélizy, France.

s Associate Professor, The University of Michigan, Ann Arbor, Michigan. 


\section{Introduction}

In local optimality, the conjugate-point concept is a key element in the development of a minimal sufficient condition (i.e., the gap between necessary and sufficient conditions is minimal) since the absence of a conjugate point on $\left[t_{0}, t_{f}\right)$ (the time interval of interest) indicates that an extremal solution is locally optimal and the presence of a conjugate point on $\left(t_{0}, t_{f}\right)$ indicates nonoptimality of a solution. In this paper, the concept of a Darboux point is introduced as an extension to global optimality of the conjugate-point concept. Such a study was motivated by several papers (Refs. 1-4) where it was noted that, in some cases, an extremal trajectory loses global optimality at a particular point. This point is given the name Darboux point in reference to Darboux's earlier recognition of this phenomenon (Ref. 5) (see also Ref. 6, p. 438), which is believed to be the first reference of this kind. An advantage of the Darboux-point concept is that it allows minimal sufficient conditions for global optimality, in the sense that the absence of a Darboux point on the interval $\left(t_{0}, t_{j}\right)$ is a necessary condition which becomes a part of a sufficient condition for the global optimality of an extremal solution when appropriately strengthened [i.e., no Darboux point on $\left.\left[t_{0}, t_{f}\right)\right]$.

A formal definition of a Darboux point is given in Section 2; theorems concerning the existence and a particular property of Darboux points are proved in Section 3; and a distinction between two types of Darboux points is made in Section 4. Finally, concluding remarks are given in Section 5. First, let us consider the following simple, but motivating, example of a Darboux point.

Example 1.1. Consider the problem of finding the minimum distance from a point to a parabola. A particular form of this problem is: minimize

$$
J=\int_{t_{0}}^{t_{f}} \sqrt{ }\left(1+u^{2}\right) d t
$$

subject to

$$
\begin{gathered}
\dot{x}=u, \quad x\left(t_{0}\right)=x_{0}, \\
x^{2}\left(t_{f}\right)+t_{f}-9=0, \quad t_{f} \leqslant 9 .
\end{gathered}
$$

The solution can be found by using the minimum principle (Ref. 7) or geometrical considerations. Consider the particular extremal trajectory $x^{*}(t)=2 t-15$ (we assume that $x_{0}=2 t_{0}-15$ ). Then (see Fig. 1), 


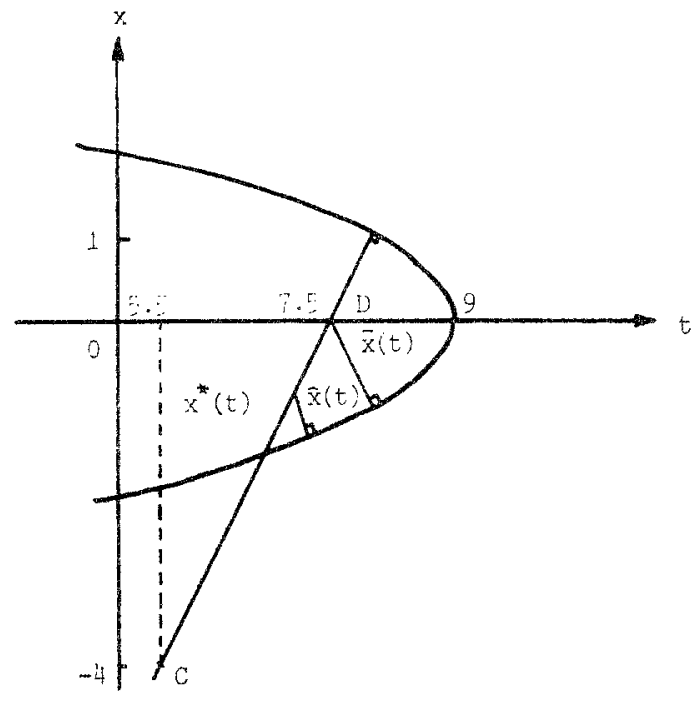

Fig. 1. Conjugate and Darboux points in the parabola problem.

(i) if $t_{0}>7.5, x^{*}(t)$ is a proper, global optimal trajectory;

(ii) if $t_{0}=7.5$, there exists another trajectory, $\bar{x}(t)$, giving the same cost as $x^{*}(t)$;

(iii) if $5.5<t_{0}<7.5$, there exists another trajectory $\hat{x}(t)$ giving a smaller cost than $x^{*}(t)$; however, $x^{*}(t)$ is still a local optimal trajectory;

(iv) if $t_{0}<5.5, x^{*}(t)$ is no longer locally optimizing.

The point $\mathrm{C}(t=5.5, x=-4)$ is a conjugate point on $x^{*}(t)$, and the point $\mathrm{D}(t=7.5, x=0)$ is a Darboux point. It is obvious from Fig. 1 that, for the example at hand, the occurrence of a Darboux point is due to the symmetry exhibited by the problem and that the line $x=0$ is a locus of Darboux points [only up to $t=8.5$, since, when $t_{0} \in(8.5,9)$, there no longer exist two distinct paths of the same length going from $x\left(t_{0}\right)=0$ to the parabola]. However, as will be seen in later examples, symmetry is not a required feature for the occurrence of a Darboux point and any general definition should not rely on symmetrical considerations, although these may aid in the characterization of a Darboux point. 


\section{Definition of a Darboux Point}

We propose the following definition for a Darboux point.

Definition 2.1. Let $x^{*}(t), t \in\left[t_{0}, t_{f}^{*}\right]$, be a trajectory which satisfies the minimum principle. A point $t_{D} \in\left[t_{0}, t_{f}^{*}\right)$ is called a Darboux point ${ }^{4}$ (see Fig. 2) if (i) for all $t_{2} \in\left(t_{D}, t_{f}^{*}\right)$, there does not exist an admissible trajectory $x(t), t \in\left[t_{2}, t_{7}\right]$, with $x\left(t_{2}\right)=x^{*}\left(t_{2}\right)$, giving a smaller value to the cost functional between $t_{2}$ and $t_{f}$ than $x^{*}(t)$ between $t_{2}$ and $t_{j}^{*}$ and if (ii) for all $t_{1} \in\left[t_{0}, t_{D}{ }^{*}\right)$, there exists an admissible trajectory $\hat{x}(t), t \in\left[t_{1}, \hat{t}_{f}\right]$, with $\hat{x}\left(t_{1}\right)=x^{*}\left(t_{1}\right)$, giving a smaller value to the cost functional between $t_{1}$ and $\hat{t}_{j}$ than $x^{*}(t)$ between $t_{1}$ and $t_{f}^{*}$.

This definition serves mainly to indicate that global optimality is lost at a Darboux point (when going backward). Implicit in the definition is that a Darboux point occurs before or at the same time as the first backward conjugate point [otherwise, (i) is not satisfied], which corresponds to the natural intuition that a trajectory loses global optimality before losing local optimality.

Remark 2.1. The backward approach used in Definition 2.1 suggests that a more precise definition would include the word backward in relation to the terminal manifold (in analogy with backward conjugate

When $t_{D}=t_{0}$, condition (ii) can be verified only if the trajectory $x *(t)$ can be extended on a small interval $\left[t_{0}-\epsilon_{,} t_{0}\right)$.

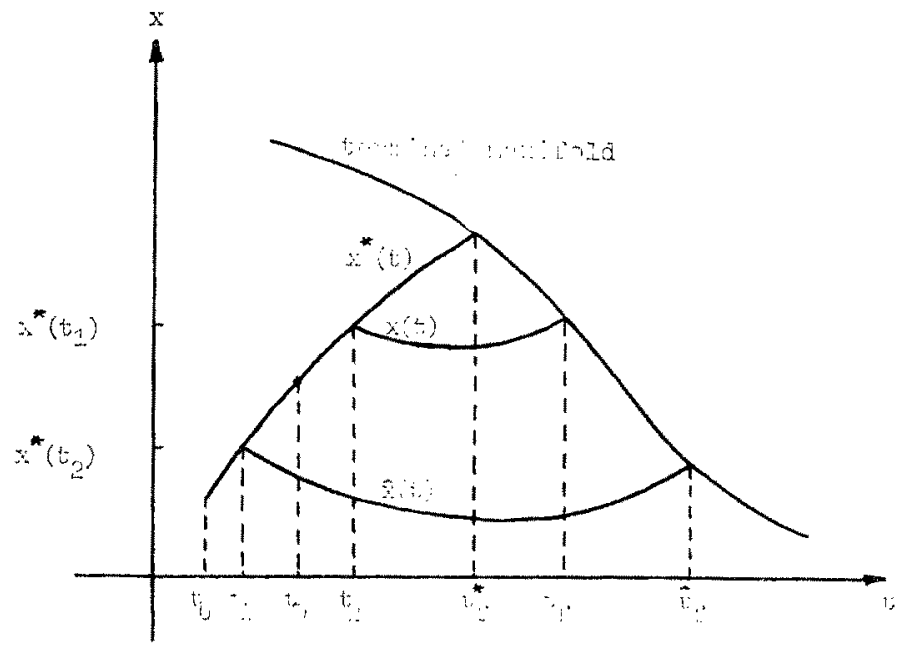

Fig. 2. The Darboux point. 
puints). Also in the case when the initial point (time and state) is not fixcd but belongs to some initial manifold, one could certainly define in a similar manner a forward Darboux point. Nevertheless, the word backward will not be included, since only fixed initial-point problems will be considered in this paper and a Darboux point will always be related to the terminal manifold.

Remark 2.2. In the definition of a conjugate point, assumptions of normality and nonsingularity are necessary because a conjugate point is defined with respect to a TPBVP, thus requiring the introduction of generalized concepts for singular problems (Ref. 8). These assumptions are not necessary in the definition of a Darboux point, and Example 2.1 below illustrates the case when a Darboux point occurs on a singular trajectory.

Example 2.1. Consider the following problem: minimize

$$
J=4 x_{f}^{4}-9 x_{f}^{3}+(11 / 2) x_{f}^{2},
$$

subject to

$$
\begin{gathered}
\dot{x}=u, \quad u \geqslant 0, \quad t \in[0,1], \\
x(0)=-1 .
\end{gathered}
$$

It can be shown that the solution

$$
u^{*}(t)=2, \quad x^{*}(t)=-1+2 t, \quad t \in[0,1],
$$

satisfies the minimum principle with the associated multiplier $\lambda^{*}(t) \equiv 0$. The trajectory $x^{*}$ is singular, and a conjugate point test is not possible. However, it can be verified directly in Fig. 3 that the value $J^{*}=0.5$ of the cost functional given by $x^{*}$ is a local minimum.

It can be proved that $t_{D}=0.75$ is a Darboux point on $x^{*}(t)$, according to Definition 2.1. Indeed, for any $t_{2} \in(0.75,1)$, a trajectory going through $x^{*}\left(t_{2}\right)$ at $t_{2}$ is given by

$$
x(t)^{*}=-1+2 t_{2}+\int_{t_{g}}^{t} u d t \geqslant-1+2 t_{2}>0.5 ;
$$

and it can be seen in Fig. 3 that $x$ cannot give a smaller value to the cost than $x^{*}$. Similarly, for any $t_{1} \in[0,0.75)$, one can choose $u=\hat{u}$ so that the corresponding trajectory $\hat{x}$ going through $x^{*}\left(t_{1}\right)$ at $t_{1}$ satisfies $\hat{x}(1) \in[0,0.5) \quad\left[\right.$ for instance, $\hat{u}=\left(3-4 t_{1}\right) / 3\left(1-t_{1}\right)$ implies that $\left.\hat{x}(1)=(2 \cdot 3) t_{1}<0.5\right]$; and it follows from Fig. 3 that $\hat{x}(t)$ gives a smaller value to the cost than $x^{*}$. 


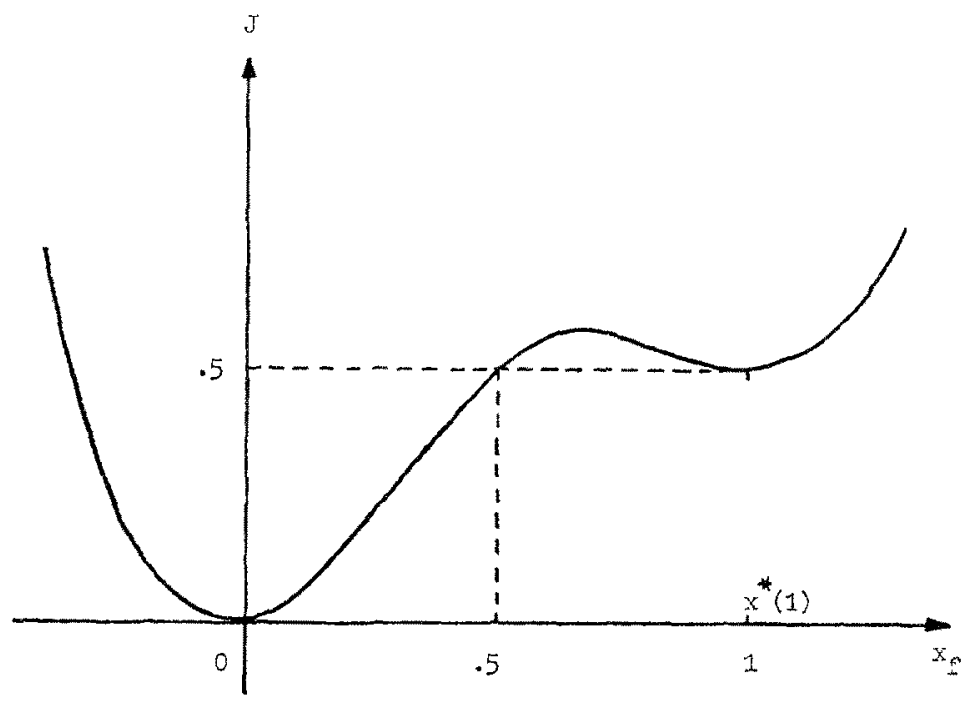

Fig. 3. Value of the cost versus $x_{f}$ in Example 2.1.

\section{Existence of a Darboux Point}

After defining the Darboux point, the next question to be considered is that of existence. Examples 1.1 and 2.1 demonstrate that a Darboux point, as defined in Section 2, exists for certain problems; and it is of interest to determine if existence is guaranteed for a general class of problems or is restricted to particular problems.

Consider an extremal trajectory $x^{*}(t), t \in\left[t_{0}, t_{f}^{*}\right]$, of some optimal control problem (details and hypotheses will be given when needed). We shall say that $\dot{x}^{*}$ is globally optimal on the interval $\left[\bar{t}, t_{i}^{*}\right], \bar{t} \in\left[t_{0}, t_{f}^{*}\right)$, if $x^{*}$ provides the cost functional with a value less than or equal to the value provided by any other admissible trajectory starting from $x^{*}(t)$ at $t=\bar{t}$. We then have the following theorem.

Theorem 3.1. If there exist $t_{1}$ and $t_{2}, t_{0} \leqslant t_{1}<t_{2}<t_{f}^{*}$, such that $x^{*}$ is globally optimal on $\left[t_{2}, t_{f}^{*}\right]$ and not globally optimal on $\left[t_{1}, t_{j}^{*}\right]$, then there exists a Darboux point $t_{D}$ on $\left(t_{1}, t_{2}\right)$.

Proof. Let $t^{\prime}$ be the middle of the interval $\left[t_{1}, t_{2}\right]$, i.e., $t^{\prime}=$ $\frac{1}{2}\left(t_{1}+t_{2}\right)$; if $x^{*}$ is globally optimal on $\left[t^{\prime}, t_{j}^{*}\right]$, keep the interval $\left[t_{1}, t^{\prime}\right]$; and, if $x^{*}$ is not globally optimal on $\left[t^{\prime}, t_{f}^{*}\right]$, keep the interval $\left[t^{\prime}, t_{2}\right]$. Let $\left[t_{1}{ }^{1}, t_{2}{ }^{1}\right]$ be the interval kept and $t^{1{ }^{\prime}}$ be the middle of $\left[t_{1}{ }^{1}, t_{2}{ }^{1}\right]$. Simi- 
larly, if $x^{*}$ is globally optimal on $\left[t^{1^{\prime}}, t_{f}^{*}\right]$, keep the interval $\left[t_{1}{ }^{1}, t^{1^{\prime}}\right]$; and, if $x^{*}$ is not globally optimal on $\left[t^{1^{\prime}}, t_{f}^{*}\right]$, keep the interval $\left[t^{1^{\prime}}, t_{2}{ }^{1}\right]$ and let $\left[t_{1}{ }^{2}, t_{2}^{2}\right]$ be the interval kept. Continuing in this way, a sequence of intervals $\left[t_{1}^{k}, t_{2}{ }^{k}\right], k=1,2, \ldots$, is generated such that $x^{*}$ is globally optimal on $\left[t_{2}{ }^{k}, t_{f}^{*}\right]$ and $x^{*}$ is not globally optimal on $\left[t_{1}{ }^{k}, t_{i}^{*}\right]$ and such that ${ }^{3}$

(i) $\left[t_{1}^{k+l}, t_{2}^{k+l}\right] \subset\left[t_{1}^{k}, t_{2}^{k}\right]$ for all $k, l$;

(ii) for any $\epsilon>0$ given, there exists $K$ such that $t_{2}^{k}-t_{1}{ }^{k}<\epsilon$ for all $k>K$;

(iii) $t_{1} \leqslant t_{1}{ }^{1} \leqslant t_{1}{ }^{2} \leqslant \cdots \leqslant t_{1}{ }^{k} \leqslant \cdots<t_{2}$;

(iv) $t_{2} \geqslant t_{2}{ }^{1} \geqslant t_{2}{ }^{2} \geqslant \cdots \geqslant t_{2}{ }^{k} \geqslant \cdots>t_{1}$;

(v) $t_{1}{ }^{k} \leqslant t_{2}{ }^{l}$ for any $k, l$.

The sequence $\left\{t_{1}^{k}\right\}$ is monotonically increasing, bounded above by $t_{2}$, and thus converges toward a limit $\bar{t}_{1}$. The sequence $\left\{t_{2}{ }^{k}\right\}$ is monotonically decreasing, bounded below by $t_{1}$, and thus converges toward a limit $\bar{t}_{2}$. Then, (v) implies that $\bar{t}_{1} \leqslant \bar{t}_{2}$; and, since $\bar{t}_{1}<\bar{t}_{2}$ would yield a contradiction with (ii), it follows that $\bar{t}_{1}=\bar{t}_{2}$. Obviously, $t_{D}=\bar{t}_{1}=\bar{t}_{2}$ satisfies Definition 2.1 [the contrary would imply contradictions with some of the properties of the sequences $\left\{t_{1}{ }^{k}\right\}$ and $\left.\left\{t_{2}^{k}\right\}\right]$, and the result follows.

So far, only times greater or less than $t_{D}$ have been considered, thus avoiding the Darboux point. However, it is of interest to know what happens at a Darboux point concerning the optimality of the trajectory $x^{*}$. We shall answer partially this question for the class of problems where the control and all the functions involved in the definition of the problem are continuous. Consider an extremal trajectory $x^{*}(t), t \in\left[t_{0}, t_{j}^{*}\right]$. We shall make as assumption somewhat related to normality. First, let us adopt the following notation: a control $u(t), t \in\left[\tau, t_{f}\right]$, is said to be admissible from $\tau$ if it is admissible (i.e., satisfying all control constraints) and if it yields an admissible trajectory (satisfying all state constraints) starting from $x^{*}(\tau)$ at $t=\tau$.

Property 3.1. If $u(t), t \in\left[\tau, t_{f}\right]$, is an admissible control from $\tau$, then, given $\epsilon>0$ small enough, there exists a control $u_{1}(t)$ admissible from $\tau_{1}$ for all $\tau_{1}$ such that $\left|\tau-\tau_{1}\right|<\epsilon$. Moreover, given $\eta>0$ arbitrarily small, there exists $\epsilon_{\eta}$ such that the distance function $\rho\left(u_{1}, u\right)<\eta$ for all $\tau_{1}$ such that $\left|\tau-\tau_{1}\right|<\epsilon_{\eta}$.

${ }^{5}$ Property (v) follows from (i) for $l>k$ and from (iv) and $t_{1}{ }^{k}<t_{2}{ }^{k}$ for $l \leqslant k$. 
Since any control considered is an element of the space $C\left[t_{0}, T_{f}\right]$ of continuous functions defined on $\left[t_{0}, T_{f}\right]$, where $t_{0}$ is the prescribed initial time and $T_{f}$ is an upper bound for the varying final time, we can choose the following distance between the controls $u(t), t \in\left[\tau, t_{f}\right]$ and $u_{1}(t), t \in\left[\tau_{x}, t_{f}{ }^{1}\right]$ :

$$
\rho\left(u_{1}, u\right)=\left|\tau-\tau_{1}\right|+\left|t_{f}-t_{f}{ }^{1}\right|+\sup _{t \in\left[t_{0} . T_{f}\right]}\left\|\hat{u}_{1}(t)-\hat{u}(t)\right\|,
$$

where $\|\cdot\|$ is the usual Euclidean norm of a finite-dimensional vector and

$$
\hat{u}(t)= \begin{cases}u(\tau) & \text { when } t \in\left[t_{0}, \tau\right], \\ u(t) & \text { when } t \in\left[\tau, t_{f}\right] \\ u\left(t_{f}\right) & \text { when } t \in\left[t_{f}, T_{f}\right]\end{cases}
$$

We make the following assumption.

Assumption 3.1. Property 3.1 holds for each $\tau \in\left[t_{0}, t_{f}^{*}\right)$.

Heuristically, Assumption 3.1 ensures that, whenever there exists an admissible trajectory with continuous control starting from any point $A$ of $x^{*}$, there exists a neighboring admissible trajectory with continuous control starting from any point of $x^{*}$ close enough to $A$. Thus, Assumption 3.1 appears as a normality condition in the class of continuous controls. We have the following theorem.

Theorem 3.2. If Assumption 3.1 is satisfied for the class of problems considered above and if there exists a Darboux point $t_{D} \in\left[t_{0}, t_{f}^{*}\right)$ on $x^{*}$, then the trajectory $x^{*}$ is globally optimal on $\left[t_{D}, t_{f}^{*}\right]$.

Proof. Any admissible trajectory starting at $x^{*}(\tau)$ when $t=\tau$ depends implicitly on $\tau$ and the choice of an admissible control $u$. Thus, the cost functional of the problem can be considered as an implicit functional $I[u, \tau]$. From the assumptions above, $I[u, \tau]$ is continuous with respect to $u$ and $\tau$. Let $t_{D}$ be a Darboux point on $x^{*}$, and suppose that $x^{*}$ is not globally optimal on $\left[t_{D}, t_{f} *\right]$; then there exists a control $\bar{u}(t)$ admissible from $t_{D}$, such that

$$
I\left[\bar{u}, t_{D}\right]<I\left[u^{*}, t_{D}\right] .
$$

From the continuity of $I$ with respect to $\tau$, for any given $\eta>0$, there exists $\epsilon_{\eta}>0$ such that

$$
\left|I\left[u^{*}, t_{D}+\epsilon\right]-I\left[u^{*}, t_{D}\right]\right|<\eta \quad \text { for all } \epsilon<\epsilon_{n} .
$$


Also, from Assumption 3.1, for any given $\delta>0$, there exists $\epsilon_{\tilde{\delta}}>0$ such that there exists a control $u_{\epsilon}(t)$ admissible from $t_{D}+\epsilon$, with

$$
\left|I\left[u_{\epsilon}, t_{D}+\epsilon\right]-I\left[\bar{u}, t_{D}\right]\right|<\delta \quad \text { for all } \epsilon<\epsilon_{\delta} .
$$

It follows from (2), (3), and (4) that $\eta$ and $\delta$ can be chosen small enough so that, for all $\epsilon<\inf \left(\epsilon_{\eta}, \epsilon_{\delta}\right)$,

$$
I\left[u_{\epsilon}, t_{D}+\epsilon\right]<I\left[u^{*}, t_{D}+\epsilon\right],
$$

which is in contradiction with part (i) of Definition 2.1. Therefore, $x^{*}$ is globally optimal on $\left[t_{D}, t_{f}^{*}\right]$.

Remark 3.1. Assumption 3.1 is sufficient to ensure the optimality of the trajectory $x^{*}$ on $\left[t_{D}, t_{f}^{*}\right]$; however, the result of Theorem 3.2 may still hold when Assumption 3.1 is not satisfied, as shown in Example 3.1 below. Example 3.1 also includes a case when Assumption 3.1 is not satisfied and Theorem 3.2 does not hold.

Example 3.1. Consider the same problem as in Example 2.1, but with the terminal constraint $\psi\left(x_{f}\right)=\sin \left(2 \pi x_{f}\right)=0$; that is, only values of the form $\frac{1}{2} k, k=0,1,2, \ldots$, are admissible for $x_{f}$. It can be shown that $x^{*}(t)=-1+2 t$ is still an extremal trajectory and that $t_{D}=0.75$ is a Darboux point. Assumption 3.1 (which was satisfied in Example 2.1)

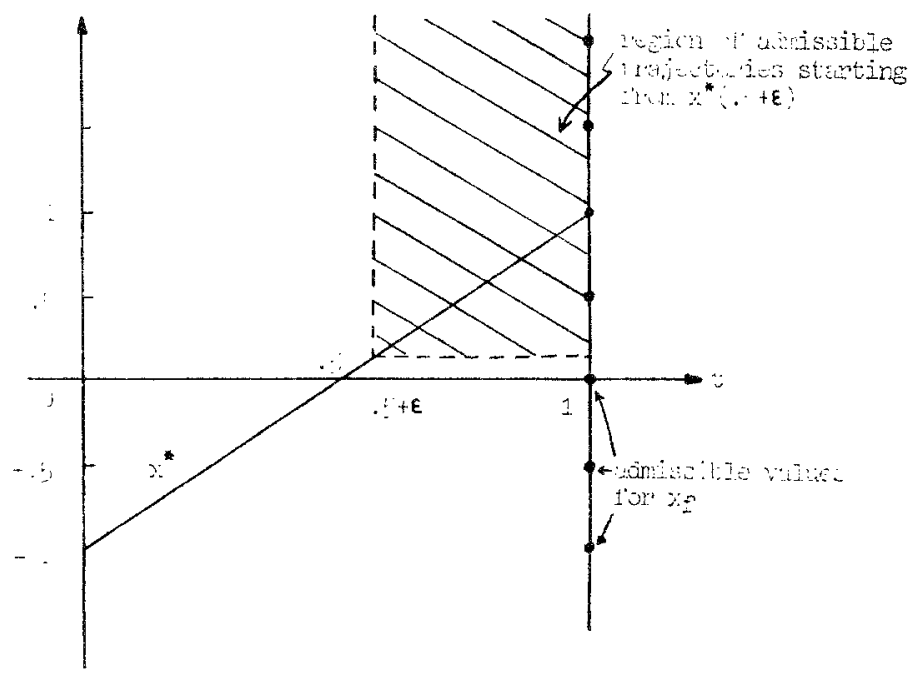

Fig. 4. Region of admissible trajectories starting from $x^{*}(0.5+\epsilon)$. 
is no longer satisfied. Indeed, for $\tau=0.5$, the control $\bar{u} \equiv 0$ is admissible from $\tau=0.5$, but there does not exist an admissible control $u$ from $\tau=0.5+\epsilon, \epsilon>0$, satisfying $\rho(u, \bar{u})<\eta$ for a given $\eta$ small enough, no matter how small $\epsilon$ is [note in Fig. 4 that the region of admissible trajectories from $x^{*}(0.5+\epsilon)$ does not include the trajectory $\left.\bar{x} \equiv 0\right]$. It can be seen from the constraints $u \geqslant 0, \psi\left(x_{f}\right)=0$ and Fig. 3 that the trajectory $x^{*}$ is globally optimal on $\left[t_{D}=0.75,1\right]$, which illustrates the first part of the remark above.

Now, consider the terminal constraint $\psi\left(x_{f}\right)=\sin \left(\pi x_{j}\right)=0$. $x_{f}=0.5$ is no longer admissible, and it can be shown that $t_{D}=0.5$ is a Darboux point on the extremal trajectory $x^{*}(t)=-1+2 t$ and that $x^{*}$ is not globally optimal on $\left[t_{D}=0.5,1\right]$, which illustrates the second part of the remark above.

\section{Darboux Points of Type 1 and Type 2}

We shall now present an example with a Darboux point somewhat different than the Darboux points of Examples 1.1 and 2.1, in the sense that a globally minimizing trajectory does not exist for the problem if $t_{0}<t_{D}$.

Example 4.1. Consider the following problem proposed by Melbourne (Ref. 3, Example B): minimize

$$
J=\int_{t_{0}}^{1} \sqrt{ }\left[v\left(1+u^{2}\right)\right] d t
$$

subject to

$$
\dot{x}=u, \quad x \geqslant 0, \quad x\left(t_{0}\right)=x_{0}, \quad x(1)=1 .
$$

An extremal trajectory is

$$
x^{*}(t)=\frac{1}{4}\left(5 t^{2}-2 t+1\right) .
$$

It can be shown that $t_{c}=0$ is a conjugate point on $x^{*}$ and that $t_{D}=0.385$ is a Darboux point (satisfying Definition 2.1).

Consider the following trajectory starting from $x^{*}(\tau)$ at $t=\tau$ (see Fig. 5):

$$
x(t, \alpha)=\left\{\begin{array}{lll}
(1 / \alpha)\left[t_{1}(\alpha)-t\right], & t \in\left[\tau, t_{1}(\alpha)\right], & t_{1}(\alpha)=\tau+\alpha x^{*}(\tau), \\
0, & t \in\left[t_{1}(\alpha), t_{2}(\alpha)\right], & t_{2}(\alpha)=1-\alpha, \\
(1 / \alpha)\left[t-t_{2}(\alpha)\right], & t \in\left[t_{2}(\alpha), 1\right], &
\end{array}\right.
$$




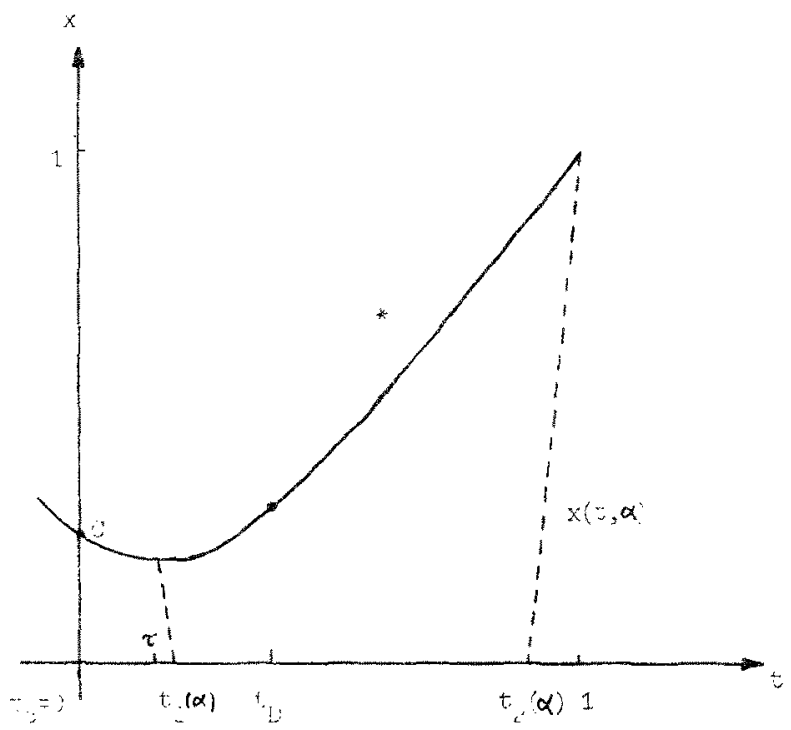

Fig. 5. Conjugate point and Darboux point in Melbourne's problem.

and let $J(x, \tau)$ be the corresponding value of the cost. By choosing $\alpha$ small enough, it can be shown that $J(\alpha, \tau)<J^{*}(\tau)$ for any $\tau<t_{D}$, where $J^{*}(\tau)$ is the value of the cost given by $x^{*}$ between $\tau$ and $t_{f}$. It was proved in Ref. 3 that, when $\tau<t_{D}$, the minimum value of $J$ is given by $x(t, \alpha=0)$, which is no longer an admissible trajectory [i.e., $x(t, \alpha=0)$ is not continuous]. Therefore, when $\tau<t_{D}, J$ does not attain its infimum over the set of admissible trajectories and the problem does not have a globally minimizing trajectory starting from $x^{*}(\tau)$ at $t=\tau$, for all $\tau<t_{D}$.

Examples 1.1, 2.1, and 4.1 demonstrate that there exist two types of Darboux points, one type indicating only the loss of global optimality for the extremal trajectory considered and another type indicating also the loss of existence of a solution to the problem. Since these two types of Darboux points are mutually exclusive, we can distinguish them in general.

Definition 4,1. Let $x^{*}(t), t \in\left[t_{0}, t_{f}^{*}\right]$, be an extremal trajectory and suppose that $t_{D} \in\left[t_{0}, t_{f}^{*}\right)$ is a Darboux point on $x^{*}$. Then, (i) if there exists an optimal trajectory to the problem starting from $x^{*}\left(t_{D}-\epsilon\right)$ at $t=t_{D}-\epsilon$ for $\epsilon>0$ arbitrarily small, $t_{D}$ is said to be a Type-l Darboux point; and (ii) if there does not exist an optimal trajectory to the problem starting from $x^{*}\left(t_{D}-\epsilon\right)$ at $t=t_{D}-\epsilon$ for $\epsilon>0$ arbitrarily small, then $t_{D}$ is said to be a Type-2 Darboux point. 
It will now be shown that Type-1 Darboux points always possess a notable property, which is not the case with Type-2 Darboux points. Consider the same class of problems as in Theorem 3.2, i.e., $x^{*}(t)$, $t \in\left[t_{0}, t_{f}^{*}\right]$, is an extremal trajectory and Assumption 3.1 is satisfied. Assume, furthermore, that $x^{*}$ has a Type-1 Darboux point $t_{D} \in\left[t_{0}, t_{f}^{*}\right)$; that is, there exists $\bar{\epsilon}>0$ such that, for all $\epsilon \in(0, \bar{\epsilon})$, there exists a control $u_{\epsilon}(t)$ admissible from $t_{D}-\epsilon$ satisfying ${ }^{6}$

$$
I\left[u_{\epsilon}, t_{D}-\epsilon\right] \leqslant I\left[u, t_{D}-\epsilon\right] \quad \text { for all admissible controls } u \text { from } t_{D}-\epsilon .
$$

Given $\bar{\epsilon}$, let $\bar{U}$ be the family of controls $u_{\epsilon}, \epsilon \in(0, \bar{\epsilon})$, defined above.

Now, let $A$ be the subset of $C\left[t_{0}, T_{f}\right]$ such that there exists $U<+\infty$ and $V<+\infty$ satisfying Properties 4.1 and 4.2 below, for all $u(t), t \in\left[\tau, t_{f}\right]$, in $A$.

Property 4.1. $\sup _{t \in\left[r, t_{f}\right]}\|u(t)\|<U$.

Property 4.2. $\left\|u\left(t_{1}\right)-u\left(t_{2}\right)\right\|<V\left|t_{1}-t_{2}\right|$ for all $t_{1}, t_{2} \in\left[\tau, t_{f}\right]$, that is, $A$ is the subset of all uniformly bounded and equilipschitzian functions on $C\left[t_{0}, T_{f}\right]$.

Let us make the following assumption. $\bar{U} \subset A$.

Assumption 4.1. There exists $\bar{\epsilon}>0$ small enough such that

Assumption 4.1 implies that there exists a smooth transition between the globally optimal trajectories of the problem starting from points on $x^{*}$ beyond (when going backward) but sufficiently close to the Type-1 Darboux point. We have the following theorem.

Theorem 4.1. If Assumptions 3.1 and 4.1 are satisfied for the class of problems considered above and if $t_{D}$ is a Type-1 Darboux point on the trajectory $x^{*}$, then at least one of the properties (i) and (ii) below is satisfied: (i) $x^{*}$ is a nonproper globally optimal trajectory on $\left[t_{D}, t_{f}^{*}\right]$, that is, there exists at least one trajectory $\bar{x}(t), t \in\left[t_{D}, \bar{t}_{f}\right]$, distinct from $x^{*}$, globally optimal on $\left[t_{D}, \bar{t}_{f}\right]$; and (ii) $t_{D}$ is a conjugate point on $x^{*}$.

Proof. Given $\bar{\epsilon}$ satisfying Assumption 4.1, choose a sequence $\left\{t_{k}\right\}$ of times $t_{k} \in\left(t_{D}-\bar{\epsilon}, t_{D}\right)$ converging toward $t_{D}$ as $k$ goes to infinity. Since $t_{D}$ is a Type-1 Darboux point, to each $t_{k}$ there corresponds a $u_{k} \in \bar{U}$. The family $\left\{u_{k}\right\}$ is defined on the submetric space $(A, \rho)$, where $\rho$

\footnotetext{
"The same notation as in the proof of Theorem 3.2 is used.
} 
is the metric defined in $(1) .(A, \rho)$ is complete and totally bounded (this follows from Properties 4.1 and 4.2; see Ref. 9, p. 139 for a proof) and therefore compact (see Ref. 9, p. 144). Also, from Assumption 4.2, the functions $u_{k}$ are uniformly bounded and equicontinuous on $(A, \rho)$ (equicontinuity follows from the equilipschitz property); and it follows that there exists a subsequence $\left\{u_{k}\right\} \subset\left\{u_{k}\right\}$ converging uniformly in the metric $p$ toward a continuous function $\tilde{u}(t), t \in\left[\tilde{t}, \tilde{t}_{f}\right]$, defined on $A$ (Ascoli's selection theorem, Ref. 10, p. 5). Since $\tilde{u}$ is the limit of elements of the subsequence $\left\{u_{k}\right\} \subset\left\{u_{k}\right\}, \tilde{t}$ is the limit of elements of a corresponding subsequence $\left\{t_{k}\right\} \subset\left\{t_{k}\right\}$, and we have $\tilde{t}=t_{D}$. Since (5) is satisfied by all $u_{k}^{l}=u_{\epsilon}$ and $u=u^{*}$,

$$
\lim _{l \rightarrow \infty} I\left[u_{k}^{l}, t_{k i}^{l}\right]=I\left[\tilde{u}, t_{D}\right] \leqslant \lim _{l \rightarrow \infty} I\left[u^{*}, t_{k}^{l}\right]=I\left[u^{*}, t_{D}\right] .
$$

We know from Theorem 3.2 that the strict inequality cannot be satisfied, and we have

$$
I\left[\tilde{u} t_{D}\right]=I\left[u^{*}, t_{D}\right] .
$$

If $\tilde{u}$ is distinct from $u^{*}$, that is, there exists $a>0$ such that $\rho\left(\tilde{u}, u^{*}\right)>a$, then (i) is proved.

If $\tilde{u}=u^{*}$ [i.e., $\forall \epsilon>0, \rho\left(\tilde{u}, u^{*}\right)<\epsilon$ ], then, for any given $\eta>0$, there exists $L_{\eta}$ such that $\rho\left(u_{k}^{l}, \tilde{u}\right)=\rho\left(u_{k}^{l}, u^{*}\right)<\eta$ for all $l>L_{\eta}$; and, from (5),

$$
I\left[u_{k}^{l}, t_{k}^{l}\right]<I\left[u^{*}, t_{k}^{l}\right]
$$

[strict inequality holds because of Definition 2.1(ii) and the definition of $\left.u_{k}^{l}\right]$. It follows that there exist trajectories $x_{k}{ }^{l}$ corresponding to $u_{k}{ }^{l}$ in any neighborhood of $x^{*}$ giving a smaller value to the cost functional than $x^{*}$. Thus, $x^{*}$ is not a locally optimizing trajectory on $\left[t_{k}{ }^{l}, t_{f}^{*}\right], l$ large enough, and there exists a conjugate point $t_{c} \in\left[t_{k}{ }^{l}, t_{D}\right]\left(t_{c} \leqslant t_{D}\right.$ follows from Definition 2.1, and $t_{e}>t_{k}{ }^{l}$ follows from the nonoptimality of $x^{*}$ on $\left.\left[t_{l_{i}}{ }^{l}, t_{f}^{*}\right]\right)$, for all $l>L_{\eta}, \eta$ small enough. Then, $t_{l}{ }^{l} \rightarrow t_{D}$ implies that $t_{c}=t_{D}$, which completes the proof of Theorem 4.1.

Comment 4.1. There does not appear to be a property similar to Theorem 4.1 for Type-2 Darboux points. Indeed, Example 4.1 is a case of a Type-2 Darboux point satisfying neither (i) nor (ii) of Theorem 4.1. Also, it can be shown that a conjugate point for a linear-quadratic problem is a Type-2 Darboux point satisfying both (i) and (ii) (Ref. 11).

Comment 4.2. Example 1.1 is a case of a Type-1 Darboux point satisfying (i) of Theorem 4.1 (this is obvious from the symmetry of the 
problem). Examples of a Type-1 Darboux point satisfying (ii) and examples of a Type-1 Darboux point satisfying both (i) and (ii) are given in Ref. 11 (Examples 5.4 and 5.5).

\section{Conclusions}

A general definition of a Darboux point was proposed and various results concerning its existence and properties were proved for a large
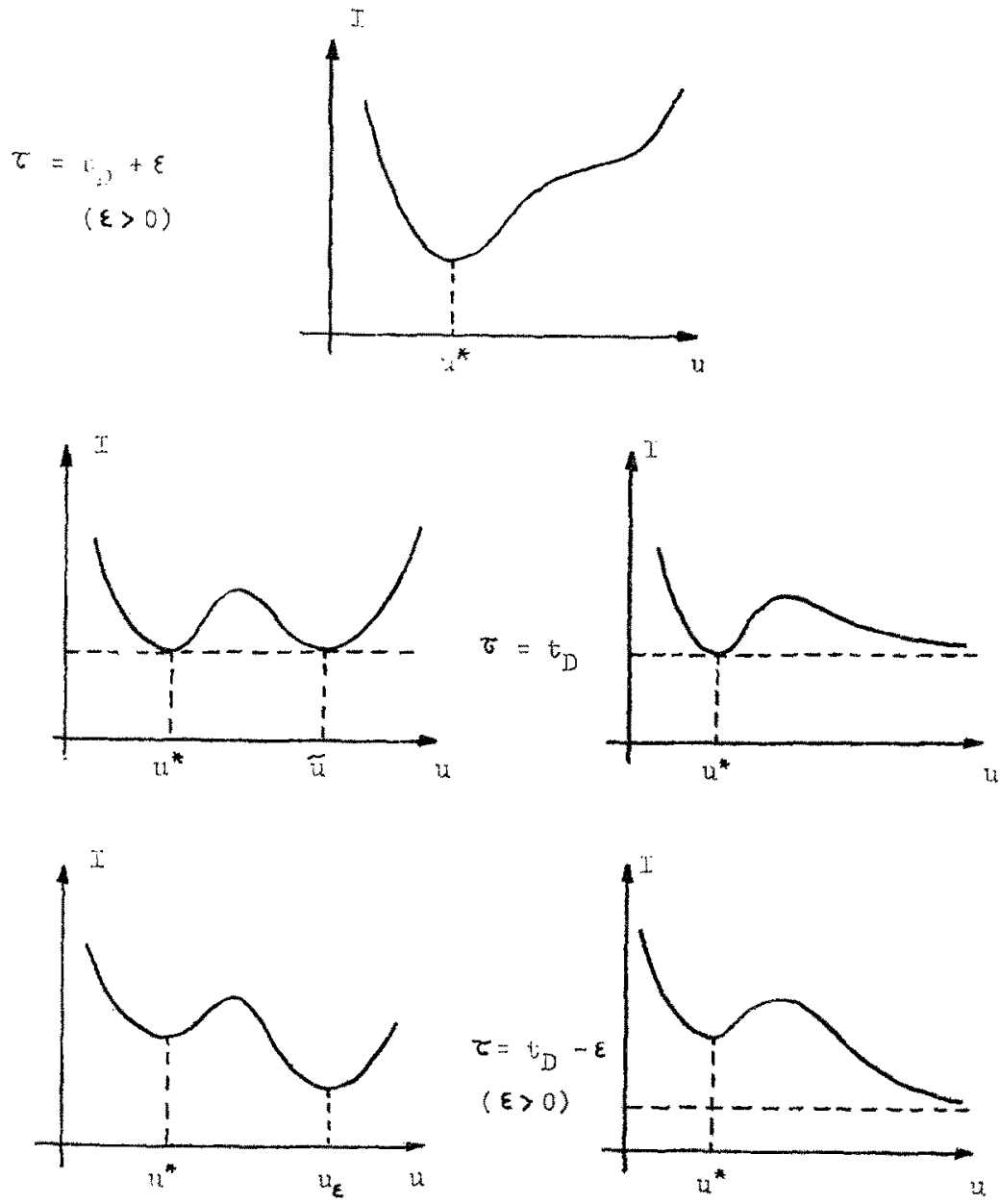

$\mathrm{T}_{1} ; 0-1$

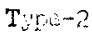

Fig. 6. Cost versus control in a neighborhood of a Darboux point. 
class of problems. A distinction between Darboux points indicating only the loss of global optimality and Darboux points indicating also the loss of existence of a global optimum was made, allowing the isolation of a property of Type-1 Darboux points. Definition 2.1, Theorems 3.2 and 4.1, and Examples 1.1, 2.1, and 4.1 allow one to develop an intuitive feel of what happens in a neighborhood of a Darboux point. Figure 6 gives several possible conceptual evolutions of the shape of the cost versus control when the initial point $\left(\tau, x^{*}(\tau)\right)$ goes back through a Darboux point $t_{D}$.

\section{References}

1. Moyer, H. G, and Kelley, H. J., Conjugate Points on Extremal Rocket Paths, Proceedings of the 19th IAF Congress, Pergamon Press, Oxford, England, 1970.

2. Kelley, H. J., Some Aspects of Two-On-One Pursuit/Evasion, Automatica, Vol. 9, pp. 403-404, 1973.

3. Melbourne, W. G., The Conjugate Point and Dynamic Programming, Jet Propulsion Laboratory, Technical Report No. 32-1043, 1966.

4. Bryson, A. E., Lecture Notes from the Summer Institute of Dynamical Astronomy, University of Texas, Austin, Texas, 1970.

5. Darboux, G., Sur la Théorie des Surfaces, Vol. III, Gauthiers-Villars, Paris, France, 1894.

6. BolzA, O., Vorlesungen über Variationsrechnung, Chelsea Publishing Company, New York, New York, 1963.

7. Bryson, A. E., and Ho, Y. C., Applied Optimal Control, Blaisdell Publishing Company, Waltham, Massachusetts, 1969.

8. MCDanell, J. P., and Powers, W. F., New Jacobi-Type Necessary and Sufficient Conditions for Singular Optimization Problems, AIAA Journal, Vol. 8, No. 8, 1970.

9. NAylor, A. W., and Sell, G. R., Linear Operator Theory in Engineering and Science, Holt, Rinehart, and Winston, New York, New York, 1971.

10. Condngton, E. A., and Levinson, N., Theory of Ordinary Differential Equations, McGraw-Hill Book Company, New York, New York, 1955.

11. Mereau, P., Global Optimality Conditions and the Darboux Point, University of Michigan, PhD Thesis, 1974. 